\title{
GEZGİN SATICI PROBLEMİNİN ÇÖZÜMÜ İÇIN MACAR ALGORİTMASI ESASLI YENİ BİR ÇÖZÜM YAKLAŞIMI
}

\author{
Kenan KARAGÜL
}

Pamukkale Üniversitesi, Honaz MYO, Yönetim ve Organizasyon Bölümü, Denizli, Türkiye

\begin{tabular}{ll}
\hline Anahtar Kelimeler & Öz \\
\hline Gezgin satıcı problemi, & Bu çalışmada kombinatoryal optimizasyon alanının ünlü problemlerinden olan \\
Macar algoritması, & gezgin satıcı ve atama problemleri arasındaki ilişkiden faydalanan yeni bir çözüm \\
Munkres algoritması, & algoritması önerilmektedir. Atama problemleri için optimal çözümü veren Macar \\
En yakın komşu sezgiseli, & Algoritması ile simetrik gezgin satıcı problemi için başlangıç çözümleri elde \\
2-Opt algoritması. & edilmiştir. Elde edilen başlangıç çözümleri En Yakın Komşu ve 2-Opt (NNH_2-Opt) \\
& sezgiselleri kullanılarak çözülmüştür. Önerilen yaklaşım sıklıkla kullanılan gezgin \\
& satıcı test problemleri ile analiz edilmiş ve bilimsel yazında yer alan bazı \\
& çalışmaların sonuçları ile klyaslama yapılmıştır. Sonuç olarak, önerilen yöntemin \\
& hem çözüm hızı hem de çözüm kalitesi bakımından kıyaslanan yöntemlere göre iyi \\
& olduğu gösterilmiştir. Özellikle, problem boyutu büyüdükçe kıyaslanan yöntemlerin \\
& çözüm süresi uzarken, önerilen yöntem büyük boyutlu problemler için de hızlı \\
& çözümler sunabilmektedir.
\end{tabular}

\section{A NOVEL SOLUTION APPROACH FOR SOLVING TRAVELING SALESMAN PROBLEM BASED ON HUNGARIAN ALGORITHM}

\begin{tabular}{l}
\hline Keywords \\
\hline Travelling salesman \\
problem, \\
Hungarian algorithm, \\
Munkres algorithm, \\
Nearest neighbor heuristic, \\
2-Opt algorithm.
\end{tabular}

\section{Keywords}

problem,

Hungarian algorithm

Nearest neighbor heuristic,

2-Opt algorithm.

\begin{abstract}
In this study, a novel solution algorithm which takes advantage of the relationship between traveling salesman and assignment problems which are famous problems of combinatorial optimization area is proposed. By using the Hungarian Algorithm, which provides the optimal solution for the assignment problems, initial solutions were obtained for the symmetric traveling salesman problem. The obtained initial solutions were solved using the Nearest Neighbor and 2-Opt (NNH_2-Opt) heuristics. The proposed approach has been analyzed with the frequently used traveling salesman test problems and compared with the results of some studies in the scientific literature. As a result, it has been shown that the proposed method is superior to the other methods with regard to solution speed and quality. In particular, as the size of the problem increases, the solution times of the compared methods are getting longer, while the proposed method can also provide fast solutions for large-scale problems.
\end{abstract}

Alıntı / Cite

Karagül, K. (2019). Gezgin Satıcı Probleminin Çözümü İçin Macar Algoritması Esaslı Yeni Bir Çözüm Yaklaşımı, Mühendislik Bilimleri ve Tasarım Dergisi, 7(3), 561-571.

\begin{tabular}{l|l}
\hline YazarKimliği / Author ID (ORCID Number) & MakaleSüreci / Article Process
\end{tabular}

\begin{tabular}{l|l}
\hline K. Karagül, 0000-0001-5397-4464 & Başvuru Tarihi / Submission Date
\end{tabular}

Revizyon Tarihi / Revision Date

Kabul Tarihi / Accepted Date

Yayım Tarihi / Published Date
07.02.2019

15.03.2019

27.03.2019

15.09.2019

\footnotetext{
* ilgili yazar / Corresponding author: kkaragul@pau.edu.tr, +90-258-811-5070
} 


\section{Giriș}

Üretilen ürünlerin, hammadde noktalarından müșterilere ulaștırılıncaya kadar var olan toplam maliyetin önemli bir mikarı lojistik operasyonlara tahsis edilmektedir. Bu nedenle ulaştırma problemleri hem teori hem uygulama açısından oldukça önemlidir. Ulaştırma problemi, $m$ adet kaynaktan $n$ adet talep noktasına ürünlerin en az maliyetle dağıtımının planlanmasında ortaya çlkan optimizasyon problemidir. Atama problemi (AP) ise $n$ adet işe atanacak $n$ adet kişinin fayda skorlarının gösterildiği bir matristen hareketle, her bir kişinin sadece tek bir işe atandığında elde edilecek en büyük faydanın belirlendiği problemdir (Kuhn, 1955; Munkres, 1957). Ulaştırma problemi ve AP birbiriyle benzer şekilde ele alınan, yakın ilișkili problemlerdir. Genel olarak bakıldığında her iki problem de kapasiteli ağ akıș probleminin özel türüdür (Dantzig ve Thapa, 1997). Diğer taraftan, yine bilimsel yazında çok çalışılan ve temel yöneylem araștırması problemlerinden biri olan gezgin satıcı problemi (GSP) yukarıda bahsedilen problemler ile büyük oranda benzerlik gösterir.

Günümüzde gerek araştırmacılar gerekse endüstriyel uygulayıcılar gezgin satıcı problemine (GSP) hızlı ve etkin çözümler üretebilecek yöntemler geliștirmek için yoğun çaba harcamaktadır. Bu çalışmada, GSP'nin çözümü için Macar Algoritması esaslı çözüm yöntemleri önerilmektedir. Ulaştırma problemi, AP ve GSP arasındaki benzerlikler üzerinden hareketle yeni çözüm yaklaşımları sunulmaya çalıșılmıştır. $\mathrm{Bu}$ bağlamda, AP için optimal çözüm üreten Macar algoritması kullanılarak GSP için başlangıç çözümleri elde edilmektedir. Bu çıtılarla GSP çözüm sezgiselleri kullanılarak GSP için hızlı ve etkin çözümler bulunması hedeflenmektedir.

Çalışmanın ikinci bölümünde Macar Algoritması ve GSP ile ilgili bilimsel yazın kısaca sunulmuştur. Üçüncü bölümde önerilen çözüm yaklaşımı, dördüncü bölümde deneysel çalışmalar ve son kısımda ise sonuç ve öneriler yer almaktadır.

\section{Bilimsel Yazın Taramas}

\subsection{Gezgin Satıcı ve Atama Problemleri İle İlgili Çalışmalar}

GSP bir satıcının belirli bir kasabadan başlayarak, satış bölgesinde yer alan tüm kasabaları sadece bir kez ziyaret etmek koşulu ile tekrar başlangıç kasabasına dönmesini ifade eder. Satıcının bu turu kapalı bir çevrimdir ve problem olası ziyaret sıralamalarının tümünü ifade eden çözüm uzayındaki en kısa turu veren çözümü elde etmektir. GSP için bir çok farklı problem tipi tanımlanmıștır. Ancak bu kapsamlı tanımlamalar yerine burada sadece uzaklık matrisine bağlı iki türü tanımlanacaktır. Uzaklık matrisine göre simetrik ve asimetrik olmak üzere iki türü vardır. Simetrik GSP için uzaklık matrisi $C=\left[c_{i j}\right]$ Her i ve j için $c_{i j}, i$ kasabasından $j$ kasabasına olan mesafe ve $c_{j i}$ tersini ifade eder ve $c_{i j}=c_{j i}$ koşulunu sağlar. Asimetrik GSP için ise $c_{i j} \neq c_{j i}$ koşulunu sağlar.

GSP tanımlanması ve anlatılması kolay, ancak çözümü oldukça zor bir problemdir. Küçük boyutlu problemler kesin matematiksel yöntemlerle çözülebilirken, çok büyük problemlerin kesin matematiksel yöntemlerle çözümü mümkün değildir. GSP bilimsel yazında NPZor sınıfında yer alan bir problem olarak belirli hale gelmiştir. Kombinatoryal optimizasyon problemleri sınıfında yer alan GSP için literatürün büyük çoğunluğu sezgisel ve metasezgisel yaklaşımlardan oluşmaktadır. Sezgisel ve metasezgisellerin yoğun șekilde kullanılması bir tesadüf değildir. Çünkü ancak bu yaklaşımların kullanımıyla optimal ve/veya optimale yakın çözümlere kabul edilebilir sürelerde ulaşlabilmektedir (Ratliff ve Rosenthal, 1983). Literatürün çok büyük bölümü GSP çözümü için sezgisel ve/veya metasezgisellere ayrıldığı için tüm literatürün ortaya konması olası değildir. Bu noktada, genetik algoritma (Zhao vd., 2009; Joines vd., 2017), akışkan genetik algoritma (Şahin ve Karagül, 2019), evrimsel hesaplamaya dayalı harmoni arama algoritması (Karagül vd., 2016), parçacık sürü optimizasyonu (Dorigo ve Gambardella, 1997), karınca kolonisi optimizasyonu(Mavrovouniotis ve Yang, 2013), tabu arama (Gendreau vd., 1998), benzetimli tavlama (Malek vd., 1989) GSP'nin çözümünde kullanılan sezgisel ve metasezgisel yöntemlere örnek olarak verilebilir.

Halim ve Ismail (2017) en yakın komşu (NN), genetik algoritma (GA), benzetimli tavlama, karınca kolonisi optimizasyonu (ACO) ve ağaç fizyolojisi optimizasyon algoritmalarının (TPO) GSP çözüm performanslarını karşılaştırmıştır. Antosiewicz vd., (2013) GSP'nin çözümü için altı adet metasezgisel yöntemi karşılaștırmıştır. Yapılan karşılaştırma sonucunda benzetimli tavlama en iyi çözümleri bulurken, tabu arama düşük varyanslı hızlı sonuçlar üretmiştir. Chitty (2017) büyük boyutlu GSP test problemlerinin çözümü için Karınca Kolonisi Optimizasyonu (ACO) yöntemini çözüm yaklaşımı olarak kullanmıştır. Önerilen yöntem ile elde edilen sonuçlar Halim ve Ismail (2017), Antosiewicz vd., (2013) ve Chitty (2017)'de yer alan sonuçlar ile çözüm süresi ve performansı bakımından kıyaslanmıştır.

GSP ve AP, uygulama ile çok yakından ilgili olmaları ve oldukça basit ve anlaşılır yapıları nedeniyle çok büyük ilgi gören klasik kombinatoryal optimizasyon problemleridir. AP için $n x n^{\prime}$ lik bir atama, $\mathrm{n}$ sayıda iş kümesinin bir permütasyonunu ifade eder. Bunun anlamı AP'nin kombinatoryal formülasyonudur ve $n$ ! içerir. GSP bir çevrime sahip permütasyon kümesine ek kısitların eklenmesi ile elde edilir. $n$ kümesinin tüm çevrimlerini içeren permütasyonlar kümesi $(n-1)$ ! elemana sahiptir. Bu bağlamda graf teorisine göre çevrimsel permütasyon bir turdur. Bir çevrim bir grafta her düğümden kesinlikle bir kez geçiyorsa 
Hamiltonyan ya da bir tur olarak adlandırılır. AP için atama politopu (İki veya daha fazla boyutta tanımlı çokgen) tüm uç noktalarının dişbükey örtüsü (convex hull) (Matematikte Dışbükey örtü veya zarf)çevrimsel permütasyonlara karşılık gelir ve GSP politopu olarak adlandırılır (Burkard, 1979). Gilmore vd. (1964), Little vd. (1963) ve Lawler (1971) tarafindan yapılan çalışmalarda atama problemi çözüm yaklaşımları ile yakın ilişkili GSP çözüm önerileri getirilmiştir. Balas ve Christofides (1981) asimetrik GSP için atama problemine dayanan çözüm yaklaşımı önermişlerdir. Lucena (1990) tarafından zamana bağlı GSP için teslim durumu analiz edilmiş ve atama ile ilişkili çözüm yaklaşımı önerilmiștir. Bir başka literatür çalışmasında Macar algoritması muğlak maliyetli GSP çözmek için kullanılmıştır (Nayak vd., 2017). Basirzadeh (2014) atama yaklaşımı ile asimetrik GSP için bir çözüm yaklaşımı önermiş ve iki küçük örnek üzerinde önerilen yöntemin çözümlerini göstermiştir. Mondal vd. (2013) tarafından yapılan çalışmada kodlar Fortran dilinde geliştirilmiş ve önerilen yaklaşım küçük problemlerle gösterilmiştir. Mondal vd. (2013) tarafından önerilen yaklaşımda yine asimetrik GSP içindir.

\subsection{Macar Algoritması İle İlgili Çalışmalar}

Macar algoritması, doğrusal programlama tekniğinin ortaya çıkışından yaklaşık 15 yıl önce D. König ve E. Egerváry adlı Macar matematikçiler tarafından önerilmiştir (Kuhn, 1955). Bu yöntem, ilgili talepler doğrultusunda homojen ürünlerin belirli kaynaklardan belirli talep noktalarına maliyetlerin en küçüklenmesini temel alarak taşınması için geliştirilmiş bir yöntemdir. İlk geliştirildiği dönemde ağ akış problemlerinin çözümünde kullanılırken, devam eden süreçte önce ulaştırma problemleri, sonrasında da doğrusal programlama problemleri için genelleștirilmiştir (Balinski ve Gomory, 1964).

AP çözümü için temel olarak iki yöntem önerilmiştir. Bunlardan birisi Kuhn'un Macar Algoritması, diğeri ise Munkres'in Macar Algoritmasıdır. Macar algoritmasının en önemli katkısı, günümüzde bütünleşik optimizasyon problemlerinin çözümü için geliştirilen algoritmalar alanındaki hızlı gelişmelerin başlangıç noktası olmasıdır (Frank, 2005).

Macar algoritmasının ulaştırma probleminin çözümüne ilişkin uzantıları literatürde yer almaktadır. Robinson (1949) asimetrik GSP'nin çözümü için Macar algoritması esaslı bir yöntem önermiştir. Balinski ve Gomory (1964) atama ve ulaştırma problemleri için iyi bilinen Macar yönteminin duali olan basit bir hesaplama yöntemi önermiştir. Bertsekas (1981) klasik atama problemi için Macar algoritmasına alternatif bir algoritma geliştirmiştir. Önerilen algoritmanın rassal olarak üretilmiş atama problemleri üzerinde yapılan testlerde üstünlük sağladığı görülmektedir. Jonker ve Volgenant (1986) Macar algoritmasını geliştirmek üzere kolay uygulanabilir üç farklı öneri sunmuştur. Yaplan geliştirmeler Macar algoritmasına göre daha etkin ve daha hızlı çözümler sağlamıştır. Kolinski ve Kolinski (2013) Macar algoritmasını örgütsel bakış açısı ile operasyonel etkinlik değerlendirmesinde kullanmıştır.

Literatür incelendiğinde çok eski zamanlara dayanan çalışmalarda GSP ve AP arasındaki ilişkilerin irdelendiği ve bazı çözüm önerileri getirildiği görülmektedir. Ancak son dönemlerde simetrik GSP'nin çözümü için önerilmiş herhangi bir çözüm yaklaşımına rastlanmamıştır. Önceki çalışmalardan farklı olarak bu çalıșmada simetrik GSP'nin çözümü üzerinde durulmaktadır. Takip eden bölümde önerilen yöntemin detayları yer almaktadır.

\section{Materyal ve Yöntem}

Çalışma kapsamında önerilen yöntem, Macar algoritması ile elde edilen çözümlerin GSP çözüm yaklaşımına (NNH_2-Opt) girdi olarak verilmesi esasına dayanmaktadır. Başka bir deyişle, kaliteli Macar algoritması çözümlerinin GSP çözüm yöntemleri için etkin başlangıç çözümleri olarak kullanılması hedeflenmiştir. Önerilen yöntemin detaylarına geçmeden önce En Yakın Komșu ve 2-Opt yöntemleri hakkında kısa bilgiler takip eden bölümlerde sunulmaktadır.

\subsection{En Yakın Komşu Sezgiseli}

En yakın komşu özellikle rota kurucu olarak görev yapan basit ama kullanışlı bir sezgiseldir. Yöntemin adımları şu şekildedir (Şahin ve Kulak, 2013);

Adım 1: Başlangıç noktasından en kısa mesafeli dağıtım noktasını belirle.

Adım 2: İlk dağıtım noktasından diğer dağıtım noktalarına olan mesafeyi belirle;

Adım 3: Mevcut mesafeler arasında en kısa olanı seç ve ikinci dağıtım noktasını belirle,

Adım 4: Tüm dağıtım noktaları tamamlanana kadar Adım 2 ve 3 ü tekrar et.

Adım 5: Dağıtım noktalarının belirlenme sırasına göre dağıtım noktalarını birleștir ve rotayı göster.

\subsection{2-0pt Sezgiseli}

Çeșitli yöntemlerle elde edilen rotaların geliștirilmesi için Croes (1958) tarafından önerilen 2-Opt yöntemi literatürde yaygın olarak kullanılmaktadır. 2-opt algoritmasının adımları aşağıda listelenmiştir (Eryavuz ve Gencer, 2001; Şahin ve Kulak, 2013);

Adım 1: Rastsal olarak turdaki parça çiftlerini belirle.

Adım 2: Tur bozulmayacak şekilde, parça çiftlerinin yerini değiștir. 
Adım 3: Yeni oluşan tur önceki tura göre bir gelişme sağlamış ise parça çiftleri yeni yerlerinde kalır, gelişme sağlanmamış ise eski yerine iade edilir.

\section{3. Önerilen Çözüm Yaklaşımları}

\subsection{1. Önerilen Yaklaşım 1: Macar-1}

Macar algoritmasına dayanan ilk çözüm yaklaşımı önerisi Macar-1 adıyla aşağıdaki şekilde önerilmiştir.

Adım 1: GSP uzaklık matrisini girdi verisi olarak al ve Macar algoritması için maliyet matrisi olarak kullan.

Adım 2: Macar algoritmasının çözümünü GSP için başlangıç çözümü olarak kullan.

Adım 3: Başlangıç çözümlerini NNH_2-Opt yaklaşımı ile çöz ve GSP sonucunu elde et.

\subsection{2. Önerilen Yaklaşım 2: Macar-4}

Macar algoritmasına dayanan ikinci çözüm yaklașımı önerisi Macar-4 adıyla aşağıdaki şekilde önerilmiștir.

Adım 1: GSP uzaklık matrisini girdi verisi olarak al.

Adım 2: GSP uzaklık matrisinin üst üçgeni dıșındaki tüm elemanlarına sonsuz değeri ata.

Adım 3: Elde edilen matrisi Macar algoritmasının maliyet matrisi olarak kullan ve çözümü bul.çözümünü GSP için başlangıç çözümü olarak kullan.Başlangıç çözümlerini NNH_2-
Opt yaklaşımı ile çöz ve GSP sonucunu elde et.

Adım 4: Macar algoritmasının çözümünü GSP için başlangıç çözümü olarak kullan.

Adım 5: Başlangıç çözümlerini NNH_2-Opt yaklaşımı ile çöz ve GSP sonucunu elde et.

Yöntemler GSP maliyet matrisinin Macar algoritmasına atama problemi matrisi olarak verilmesi ile başlar ve atama çözümü GSP çözüm algoritması için bașlangıç çözümü olarak belirlenir. Bu noktada iki farklı yaklaşım geliştirilmiştir. Birinci yaklaşımda, standart GSP maliyet matrisi Macar algoritmasına girdi olarak verilmiș ve Macar-1 olarak adlandırılmıștır. İkinci yaklașımda ise GSP maliyet matrisinin alt üçgeni olarak ifade edilen kısmının sonsuz değerler atanması ile elde edilen matris Macar algoritması için girdi olarak verilmiş ve bu yöntem ise Macar-4 olarak adlandırılmıştır. Önerilen yaklaşımlar Ek-1'de koordinatları ve uzaklık matrisi verilen küçük bir problem üzerinde açıklanmıştır. Örnek problem Antosiewicz vd., (2013) tarafından GSP için üretilmiş en küçük boyutlu iki problemden biridir. Uzaklık matrisleri ve önerilen çözüm yöntemi ile elde edilen çözümler sırasıyla Tablo 1 ve Tablo 2'de sunulmuştur. Tablo 1'de Macar-1 yaklaşımının girdi matrisi ve Macar algoritmasının GSP çözümü (1062) ile NNH_2Opt yaklaşımının çözümü (426) gösterilmiştir. Tablo 2 'de ise Macar-4 yaklaşımı için kullanılan girdi matrisi, Macar algoritması ile elde edilen GSP çözümü (1062) ve NNH_2-Opt yaklaşımının çözümü (397) yer almaktadır. Elde edilen 397 değeri aynı zamanda bu problem için optimal çözüm değeridir.

Tablo 1. GSP Çözümü İçin Macar-1 Girdi Matrisi

\begin{tabular}{|c|c|c|c|c|c|c|c|c|c|c|c|c|c|c|c|c|c|c|c|c|}
\hline & 1 & 2 & 3 & 4 & 5 & 6 & 7 & 8 & 9 & 10 & 11 & 12 & 13 & 14 & 15 & 16 & 17 & 18 & 19 & 20 \\
\hline 1 & 0 & 84 & 51 & 81 & 111 & 68 & 31 & 56 & 117 & 48 & 62 & 69 & 80 & 90 & 26 & 55 & 44 & 71 & 69 & 60 \\
\hline 2 & 84 & 0 & 60 & 23 & 28 & 65 & 56 & 35 & 34 & 38 & 22 & 17 & 52 & 6 & 67 & 29 & 44 & 18 & 52 & 66 \\
\hline 3 & 51 & 60 & 0 & 72 & 87 & 88 & 26 & 55 & 85 & 45 & 48 & 54 & 88 & 66 & 56 & 43 & 23 & 58 & 19 & 83 \\
\hline 4 & 81 & 23 & 72 & 0 & 35 & 45 & 59 & 25 & 49 & 34 & 24 & 18 & 29 & 24 & 59 & 30 & 51 & 14 & 69 & 47 \\
\hline 5 & 111 & 28 & 87 & 35 & 0 & 79 & 84 & 58 & 19 & 64 & 49 & 42 & 61 & 22 & 92 & 56 & 73 & 41 & 77 & 82 \\
\hline 6 & 68 & 65 & 88 & 45 & 79 & 0 & 64 & 36 & 94 & 44 & 51 & 51 & 20 & 68 & 42 & 50 & 66 & 48 & 95 & 8 \\
\hline 7 & 31 & 56 & 26 & 59 & 84 & 64 & 0 & 36 & 88 & 26 & 36 & 44 & 68 & 62 & 30 & 29 & 13 & 46 & 41 & 58 \\
\hline 8 & 56 & 35 & 55 & 25 & 58 & 36 & 36 & 0 & 68 & 11 & 15 & 18 & 33 & 40 & 34 & 14 & 32 & 18 & 59 & 34 \\
\hline 9 & 117 & 34 & 85 & 49 & 19 & 94 & 88 & 68 & 0 & 71 & 56 & 50 & 77 & 28 & 101 & 63 & 75 & 50 & 71 & 96 \\
\hline 10 & 48 & 38 & 45 & 34 & 64 & 44 & 26 & 11 & 71 & 0 & 15 & 22 & 44 & 43 & 30 & 9 & 22 & 23 & 51 & 40 \\
\hline 11 & 62 & 22 & 48 & 24 & 49 & 51 & 36 & 15 & 56 & 15 & 0 & 7 & 44 & 28 & 45 & 8 & 27 & 11 & 47 & 49 \\
\hline 12 & 69 & 17 & 54 & 18 & 42 & 51 & 44 & 18 & 50 & 22 & 7 & 0 & 41 & 22 & 51 & 15 & 34 & 4 & 52 & 50 \\
\hline 13 & 80 & 52 & 88 & 29 & 61 & 20 & 68 & 33 & 77 & 44 & 44 & 41 & 0 & 53 & 54 & 46 & 66 & 38 & 91 & 26 \\
\hline 14 & 90 & 6 & 66 & 24 & 22 & 68 & 62 & 40 & 28 & 43 & 28 & 22 & 53 & 0 & 73 & 35 & 51 & 22 & 57 & 69 \\
\hline 15 & 26 & 67 & 56 & 59 & 92 & 42 & 30 & 34 & 101 & 30 & 45 & 51 & 54 & 73 & 0 & 38 & 39 & 51 & 70 & 35 \\
\hline 16 & 55 & 29 & 43 & 30 & 56 & 50 & 29 & 14 & 63 & 9 & 8 & 15 & 46 & 35 & 38 & 0 & 21 & 17 & 45 & 47 \\
\hline 17 & 44 & 44 & 23 & 51 & 73 & 66 & 13 & 32 & 75 & 22 & 27 & 34 & 66 & 51 & 39 & 21 & 0 & 38 & 31 & 61 \\
\hline 18 & 71 & 18 & 58 & 14 & 41 & 48 & 46 & 18 & 50 & 23 & 11 & 4 & 38 & 22 & 51 & 17 & 38 & 0 & 56 & 48 \\
\hline 19 & 69 & 52 & 19 & 69 & 77 & 95 & 41 & 59 & 71 & 51 & 47 & 52 & 91 & 57 & 70 & 45 & 31 & 56 & 0 & 91 \\
\hline 20 & 60 & 66 & 83 & 47 & 82 & 8 & 58 & 34 & 96 & 40 & 49 & 50 & 26 & 69 & 35 & 47 & 61 & 48 & 91 & 0 \\
\hline
\end{tabular}

[ GSP İçin Macar-1 Çözümü ]

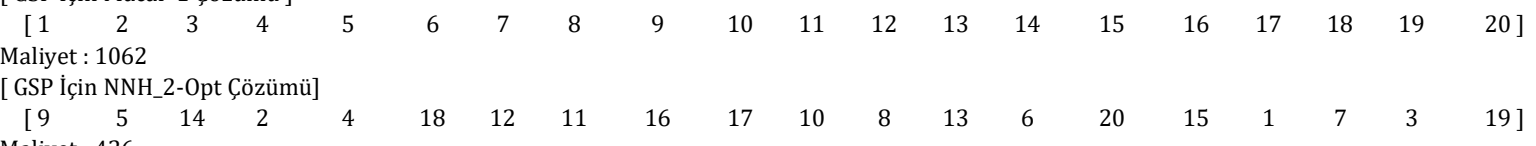

Maliyet : 426 
Tablo 2. GSP Çözümü İçin Macar-4 Girdi Matrisi

\begin{tabular}{|c|c|c|c|c|c|c|c|c|c|c|c|c|c|c|c|c|c|c|c|c|}
\hline & 1 & 2 & 3 & 4 & 5 & 6 & 7 & 8 & 9 & 10 & 11 & 12 & 13 & 14 & 15 & 16 & 17 & 18 & 19 & 20 \\
\hline 1 & Inf & 84 & 51 & 81 & 111 & 68 & 31 & 56 & 117 & 48 & 62 & 69 & 80 & 90 & 26 & 55 & 44 & 71 & 69 & 60 \\
\hline 2 & Inf & Inf & 120 & 46 & 56 & 130 & 112 & 70 & 68 & 76 & 44 & 34 & 104 & 12 & 134 & 58 & 88 & 36 & 104 & 132 \\
\hline 3 & Inf & Inf & Inf & 144 & 174 & 176 & 52 & 110 & 170 & 90 & 96 & 108 & 176 & 132 & 112 & 86 & 46 & 116 & 38 & 166 \\
\hline 4 & Inf & Inf & Inf & Inf & 70 & 90 & 118 & 50 & 98 & 68 & 48 & 36 & 58 & 48 & 118 & 60 & 102 & 28 & 138 & 94 \\
\hline 5 & Inf & Inf & Inf & Inf & Inf & 158 & 168 & 116 & 38 & 128 & 98 & 84 & 122 & 44 & 184 & 112 & 146 & 82 & 154 & 164 \\
\hline 6 & Inf & Inf & Inf & Inf & Inf & Inf & 128 & 72 & 188 & 88 & 102 & 102 & 40 & 136 & 84 & 100 & 132 & 96 & 190 & 16 \\
\hline 7 & Inf & Inf & Inf & Inf & Inf & Inf & Inf & 72 & 176 & 52 & 72 & 88 & 136 & 124 & 60 & 58 & 26 & 92 & 82 & 116 \\
\hline 8 & Inf & Inf & Inf & Inf & Inf & Inf & Inf & Inf & 136 & 22 & 30 & 36 & 66 & 80 & 68 & 28 & 64 & 36 & 118 & 68 \\
\hline 9 & Inf & Inf & Inf & Inf & Inf & Inf & Inf & Inf & Inf & 142 & 112 & 100 & 154 & 56 & 202 & 126 & 150 & 100 & 142 & 192 \\
\hline 10 & Inf & Inf & Inf & Inf & Inf & Inf & Inf & Inf & Inf & Inf & 30 & 44 & 88 & 86 & 60 & 18 & 44 & 46 & 102 & 80 \\
\hline 11 & Inf & Inf & Inf & Inf & Inf & Inf & Inf & Inf & Inf & Inf & Inf & 14 & 88 & 56 & 90 & 16 & 54 & 22 & 94 & 98 \\
\hline 12 & Inf & Inf & Inf & Inf & Inf & Inf & Inf & Inf & Inf & Inf & Inf & Inf & 82 & 44 & 102 & 30 & 68 & 8 & 104 & 100 \\
\hline 13 & Inf & Inf & Inf & Inf & Inf & Inf & Inf & Inf & Inf & Inf & Inf & Inf & Inf & 106 & 108 & 92 & 132 & 76 & 182 & 52 \\
\hline 14 & Inf & Inf & Inf & Inf & Inf & Inf & Inf & Inf & Inf & Inf & Inf & Inf & Inf & Inf & 146 & 70 & 102 & 44 & 114 & 138 \\
\hline 15 & Inf & Inf & Inf & Inf & Inf & Inf & Inf & Inf & Inf & Inf & Inf & Inf & Inf & Inf & Inf & 76 & 78 & 102 & 140 & 70 \\
\hline 16 & Inf & Inf & Inf & Inf & Inf & Inf & Inf & Inf & Inf & Inf & Inf & Inf & Inf & Inf & Inf & Inf & 42 & 34 & 90 & 94 \\
\hline 17 & Inf & Inf & Inf & Inf & Inf & Inf & Inf & Inf & Inf & Inf & Inf & Inf & Inf & Inf & Inf & Inf & Inf & 76 & 62 & 122 \\
\hline 18 & Inf & Inf & Inf & Inf & Inf & Inf & Inf & Inf & Inf & Inf & Inf & Inf & Inf & Inf & Inf & Inf & Inf & Inf & 112 & 96 \\
\hline 19 & Inf & Inf & Inf & Inf & Inf & Inf & Inf & Inf & Inf & Inf & Inf & Inf & Inf & Inf & Inf & Inf & Inf & Inf & Inf & 182 \\
\hline 20 & Inf & Inf & Inf & Inf & Inf & Inf & Inf & Inf & Inf & Inf & Inf & Inf & Inf & Inf & Inf & Inf & Inf & Inf & Inf & Inf \\
\hline \multicolumn{21}{|c|}{ [ GSP İçin Macar-4 Çözümü ] } \\
\hline & {$[2$} & 3 & 4 & 5 & 6 & 7 & 8 & 9 & 10 & 11 & 12 & 13 & 14 & 15 & 16 & 17 & 18 & 19 & 20 & 1] \\
\hline & \multicolumn{20}{|c|}{ Maliyet : 1062} \\
\hline & {$[4$} & 5 & 9 & 14 & 2 & 18 & 12 & 11 & 16 & 8 & 10 & 17 & 19 & 3 & 7 & 1 & 15 & 20 & 6 & $13]$ \\
\hline
\end{tabular}

\section{Araştırma Bulguları}

\subsection{Deneysel Sonuçlar}

Yapılan ön değerlendirmeler sonucunda Macar-1 algoritmasının etkin çözümler üretemediği gözlemlenmiştir. $\mathrm{Bu}$ nedenle Macar-4 yaklaşımı ile literatürdeki GSP çözümleri arasındaki durum değerlendirmeye alınmıștır. Yapılan tüm analizlerde Windows 10 ișletim sistemi üzerinde Matlab 2016b ve Intel Core i7-4800MQ, $2.70 \mathrm{GHz}, 16 \mathrm{MB}$ dizüstü bilgisayar tek çekirdek ile kullanılmıștır. Bu amaçla ilk aşamada Antosiewicz vd., (2013) tarafından kullanılan sekiz adet GSP problemi analiz edilmiş ve bir durum değerlendirmesi yapılmıștır. Karşılaştırmalar Tablo 3'te sunulmuştur. Bu tabloda sekiz adet problemin Test, OPT, GA, HS, PSO, QA, SA, TS, 2-OPT, Macar-4 başlıkları sırası ile problem adı, Optimal çözüm, Genetik Algoritma, Harmonik Arama, Parçacık Sürü Optimizasyonu, Kuantum Algoritması, Benzetimli Tavlama, Tabu Arama, 2_0pt ve Macar-4 girdisine dayanan NNH-2_Opt çözümlerini göstermektedir. Bu tablodaki sezgisel çözümler ve optimal çözümler Antosiewicz vd. (2013) makalesinden alınmıştır.

Tablo 3. Küçük Boyutlu GSP Çözümleri Karşılaştırması

\begin{tabular}{|c|c|c|c|c|c|c|c|c|c|}
\hline Test & OPT & GA & HS & PSO & QA & SA & TS & 2-0PT & Macar-4 \\
\hline $20(\mathrm{a})$ & $\mathbf{3 9 7}$ & 510 & 524 & 544 & 480 & 408 & 430 & 524 & 397 \\
\hline $20(\mathrm{~b})$ & $\mathbf{3 6 7}$ & 535 & 403 & 556 & 494 & 367 & 436 & 553 & 367 \\
\hline $50(\mathrm{a})$ & $\mathbf{5 6 0}$ & 1613 & 1109 & 1790 & 1041 & 586 & 703 & 996 & 592 \\
\hline $50(\mathrm{~b})$ & $\mathbf{5 7 1}$ & 1576 & 1116 & 1746 & 1008 & 695 & 700 & 1011 & 587 \\
\hline $80(\mathrm{a})$ & $\mathbf{7 0 9}$ & 2693 & 2446 & 2931 & 2154 & 802 & 909 & 1325 & 723 \\
\hline $80(\mathrm{~b})$ & $\mathbf{6 8 7}$ & 2812 & 2541 & 2098 & 2273 & 779 & 903 & 1280 & 703 \\
\hline att48 & $\mathbf{3 3 3}$ & 398 & 524 & 883 & 485 & 342 & 385 & 570 & 350 \\
\hline eil76 & $\mathbf{5 3 8}$ & 785 & 1284 & 1783 & 1170 & 582 & 642 & 887 & 558 \\
\hline Ortalama & $\mathbf{5 2 0 , 2 5}$ & $\mathbf{1 3 6 5 , 2 5}$ & $\mathbf{1 2 4 3 , 3 8}$ & $\mathbf{1 5 4 1 , 3 8}$ & $\mathbf{1 1 3 8 , 1 3}$ & $\mathbf{5 7 0 , 1 3}$ & $\mathbf{6 3 8 , 5 0}$ & $\mathbf{8 9 3 , 2 5}$ & $\mathbf{5 3 4 , 6 3}$ \\
\hline
\end{tabular}

Tablo 3 incelendiğinde ortalama çözüm değerleri tüm problemler için optimal ortalama değeri 520,25'ten oldukça uzaktadır. En yakın ortalama değer 534 ile Macar-4 yaklaşımına aittir. Macar-4 yaklaşımı 1 ve 2 nolu problemlerde optimal değere ulaşırken, optimalden sapma ortalaması $\% 2,76$ olarak gerçekleşmiştir. Aynı zamanda çözüm süreleri açısından bir karşılaştırma yapıldığında her bir problem 100 sn çalıştırılarak sezgisel algoritmaların çözümleri elde edilmiştir. Macar-4 yaklaşımına dayanan GSP çözümü 20 (a) için 0,0652 sn ve 80 (b) problemi için 0,0914 sn ve 100 sn ile kıyaslanamayacak kadar küçüktür. 
Tablo 4. GSP Çözümlerinin Karşılaştırması

\begin{tabular}{|c|c|c|c|c|c|c|c|c|c|c|c|c|c|c|}
\hline & & \multicolumn{11}{|c|}{ NNH-2_Opt } & \multirow[b]{2}{*}{ NN } & \multirow[b]{2}{*}{ Macar-4 } \\
\hline Test & OPT & 1 & 2 & 3 & 4 & 5 & 6 & 7 & 8 & 9 & 10 & Ort & & \\
\hline 20 (a) & 397 & 397 & 397 & 404 & 438 & 402 & 402 & 401 & 404 & 401 & 397 & 404,3 & 406,5 & 397 \\
\hline 20 (b) & 367 & 367 & 367 & 367 & 367 & 367 & 367 & 367 & 367 & 367 & 367 & 367,0 & 380,0 & 367 \\
\hline 50 (a) & 560 & 593 & 588 & 602 & 584 & 600 & 598 & 574 & 612 & 570 & 592 & 591,3 & 656,1 & 592 \\
\hline $50(\mathrm{~b})$ & 571 & 597 & 587 & 612 & 589 & 580 & 597 & 582 & 602 & 595 & 587 & 592,8 & 615,2 & 587 \\
\hline 80 (a) & 709 & 722 & 736 & 764 & 729 & 739 & 741 & 716 & 719 & 728 & 737 & 733,1 & 797,4 & 723 \\
\hline $80(\mathrm{~b})$ & 687 & 700 & 742 & 708 & 694 & 699 & 697 & 726 & 716 & 726 & 703 & 711,1 & 721,4 & 703 \\
\hline att48 & 333 & 345 & 350 & 342 & 350 & 345 & 350 & 352 & 337 & 348 & 362 & 348,1 & 392,4 & 350 \\
\hline eil76 & 538 & 555 & 546 & 567 & 563 & 564 & 552 & 567 & 559 & 565 & 555 & 559,3 & 612,7 & 558 \\
\hline Ortalama & 520,25 & 534,50 & 539,13 & 545,75 & 539,25 & 537,00 & 538,00 & 535,63 & 539,50 & 537,50 & 537,50 & 538,38 & 572,7 & 534,63 \\
\hline
\end{tabular}

Tablo 4'te sekiz adet küçük GSP problemi için NNH_2Opt her problem için on kez koșturulmuș ve Ort sütununda bu koşturmalara ilişkin ortalama değerler gösterilmiştir. NN olarak adlandırılan sütunda ise En Yakın komşu algoritmasına ilişkin çözümler gösterilmiştir. Son sütunda yine Macar-4 yaklaşımına dayanan NNH_2-Opt çözümleri gösterilmiștir. Ortalamalar üzerinden bakıldığında NNH_2-Opt yaklaşımının ve standart NN yaklaşımlarının daha kötü çözümler ürettiği görülmektedir. Ancak elde edilen çözümlere bakıldığında NN yaklaşımının en kötü çözümleri verdiği, NNH_2-Opt yaklaşımın optimal çözümleri yakaladığı görülmektedir.

Tablo 5'te Halim ve Ismail (2017)'de yer alan NN, GA, SA, TS, ACO, TPO sezgisel algoritmaları ile TSPLIB (Traveling Salesman Problem Library, Literatürde en çok kullanılan GSP test problemleri kütüphanesi)'deki bir grup problem analiz edilmiştir. Bu makalede elde edilen araştırma sonuçları Macar-4 algoritmasına dayanan GSP çözümleri ile çözüm kalitesi ve süre açısından karşılaştırılmıştır. Deneyler için kullanılan veri setleri Heidelber Üniversitesi web sayfasından alınmıștır (TSPLIB95, 2018).

Tablo 5 incelendiğinde, Macar-4 yönteminin çözüm süreleri rd400 problemi dışındaki tüm problemler için 1 sn'den daha küçüktür. Diğer yöntemlerin çözüm süreleri ise ilgili çalışmada yer alan süre grafikleri üzerinden yapılan değerlendirmeye göre oldukça uzundur.

Tablo 5'teki problemlerinin çözümlerine ilişkin göreli karşılaştırmalar Tablo 6'da sunulmuştur. Her bir yaklaşımdan elde edilen ortalama çözüm değeri optimal çözümlere oranlanmıştır. Hem optimal çözüme göre hem de çözümler arasındaki göreli üstünlük bu sayede açıkça görülmektedir. Bağıl oran sütunundaki 1 değeri optimal çözümü, 1'den büyük değerler ise optimale yaklaşımı ifade etmektedir.

Tablo 6 incelendiğinde ilk sütundaki problemler için Macar-4 yaklașımına dayanan GSP çözümü göreli olarak kötü ancak rekabetçidir. Ancak ikinci sütunda yer alan çözümlerde Macar-4 yaklaşımının çözüm üstünlüğü açıkça görülmektedir. Sonuç olarak, problem boyutunun büyümesiyle birlikte önerilen yöntem diğer yöntemlere kıyasla hem süre hem de kalite bakımından oldukça iyi sonuçlar sağlamaktadır. Önerilen yöntemin büyük boyutlu problemlerin çözümündeki performansını değerlendirmek için Chitty (2017) tarafından kullanılan veri setlerinden yararlanılmıştır. 5 adet büyük veri setinin Macar-4 yöntemiyle elde edilen çözüm değerleri ve süreleri Karınca Kolonisi Optimizasyon Algoritması ile edilen sonuçlar ile kıyaslanmıştır. Elde edilen sonuçlar Tablo 7'de gösterilmektedir.

Tablo 6 incelendiğinde ortalama çözüm kaliteleri açısından Karınca Kolonisi Optimizasyon Algoritmasının daha iyi bir performans sergilediği görülmektedir. Ancak yine problem boyutu büyüdükçe çözüm kalitesi üstünlüğü Macar-4 temelli GSP çözüm yaklaşımına geçmektedir. Bir diğer boyut olan çözüm süreleri incelendiğinde Macar-4 yönteminin tartışmasız olarak çok üstün durumda olduğu görülmektedir.

\section{Sonuç ve Tartışma}

$\mathrm{Bu}$ çalışma da gezgin satıcı problemini çözmek için Macar algoritmasına dayanan çözüm yaklaşımları önerilmiștir. Calıșmanın temeli ${ }_{2}$ atama problemlerini optimal olarak çözen Macar algoritmasına dayanmaktadır. Buradan hareketle GSP probleminin çözümü için bir katkı oluşturulabilir mi sorusu araştırılmıștır. Yapılan araştırma GSP uzaklık matrisinin standart girdi olarak Macar algoritmasına verilmesi ile elde edilen atamanın GSP için iyi bir çözüm elde edilip edilmediği analiz edilmiştir. Görülmüştür ki atama problemi için optimal çözümler üreten Macar algoritması GSP için iyi çözümler üretememektedir. Bunun üzerine GSP maliyet matrisinde küçük değişiklikler yapılarak daha iyi çözümler üretilip üretilemediği araştırılmış ancak çözümlerin yine optimal GSP çözümüne çok uzak olduğu görülmüştür. Ancak Macar-4 yaklașımı olarak adlandırılan uzaklık matrisi üzerinde yapılan değişikliklerin GSP için iyi başlangıç çözümleri olduğu görülmüștür. Bu bağlamda yapılan analizler ile Macar4 yaklaşımı ile elde edilen çıktıların En Yakın Komşu ve 2-Opt yaklaşımlarını birlikte kullanan hibrit yaklaşım ile oldukça iyi sonuçlar verdiği gözlemlenmiştir. Bu gözlemler bilimsel yazında yer alan GSP test problemleri ile yapılan analizler sonucunda da doğrulanmıștır. 
Tablo 5. GSP Test Problemleri Karşılaştırması

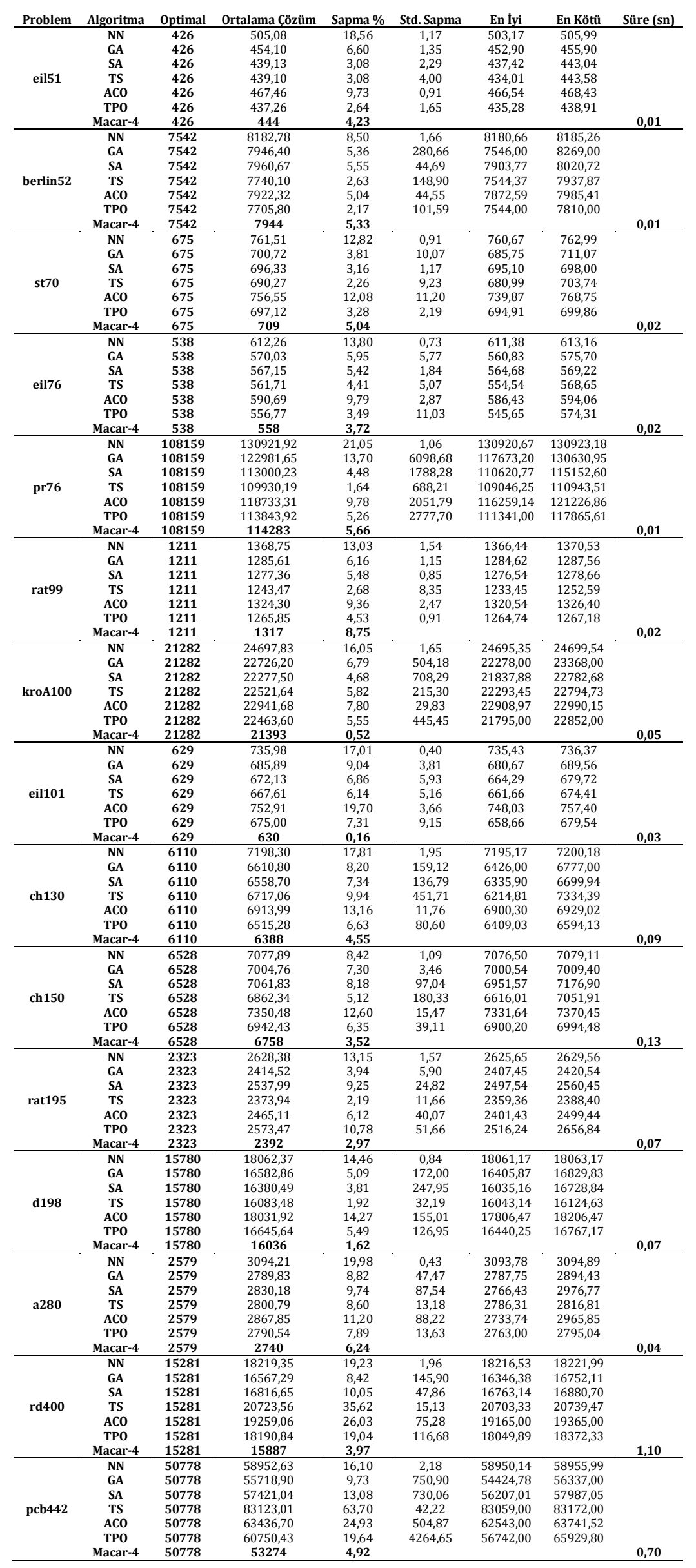


Tablo 6. GSP Test Problemleri Göreli Karşılaştırması

\begin{tabular}{|c|c|c|c|c|c|}
\hline Problem & Algoritma & Bağıl Oran & Problem & Algoritma & Bağıl Oran \\
\hline \multirow{7}{*}{ eil51 } & NN & 1,19 & \multirow{7}{*}{$\operatorname{ch} 130$} & NN & 1,18 \\
\hline & GA & 1,07 & & GA & 1,08 \\
\hline & SA & 1,03 & & SA & 1,07 \\
\hline & TS & 1,03 & & TS & 1,10 \\
\hline & ACO & 1,10 & & ACO & 1,13 \\
\hline & TPO & 1,03 & & TPO & 1,07 \\
\hline & Macar-4 & 1,04 & & Macar-4 & 1,05 \\
\hline \multirow{7}{*}{ berlin52 } & NN & 1,08 & \multirow{7}{*}{$\operatorname{ch} 150$} & $\mathrm{NN}$ & 1,08 \\
\hline & GA & 1,05 & & GA & 1,07 \\
\hline & SA & 1,06 & & SA & 1,08 \\
\hline & TS & 1,03 & & TS & 1,05 \\
\hline & ACO & 1,05 & & ACO & 1,13 \\
\hline & TPO & 1,02 & & TPO & 1,06 \\
\hline & Macar-4 & 1,05 & & Macar-4 & 1,04 \\
\hline \multirow{7}{*}{ st70 } & NN & 1,13 & \multirow{7}{*}{ rat195 } & NN & 1,13 \\
\hline & GA & 1,04 & & GA & 1,04 \\
\hline & SA & 1,03 & & SA & 1,09 \\
\hline & TS & 1,02 & & TS & 1,02 \\
\hline & $\mathrm{ACO}$ & 1,12 & & $\mathrm{ACO}$ & 1,06 \\
\hline & TPO & 1,03 & & TPO & 1,11 \\
\hline & Macar-4 & 1,05 & & Macar-4 & 1,03 \\
\hline \multirow{7}{*}{ eil76 } & NN & 1,14 & \multirow{7}{*}{ d198 } & NN & 1,14 \\
\hline & GA & 1,06 & & GA & 1,05 \\
\hline & SA & 1,05 & & SA & 1,04 \\
\hline & TS & 1,04 & & TS & 1,02 \\
\hline & ACO & 1,10 & & ACO & 1,14 \\
\hline & TPO & 1,03 & & TPO & 1,05 \\
\hline & Macar-4 & 1,04 & & Macar-4 & 1,02 \\
\hline \multirow{7}{*}{ pr76 } & NN & 1,21 & \multirow{7}{*}{ a280 } & $\mathrm{NN}$ & 1,20 \\
\hline & GA & 1,14 & & GA & 1,08 \\
\hline & SA & 1,04 & & SA & 1,10 \\
\hline & TS & 1,02 & & TS & 1,09 \\
\hline & ACO & 1,10 & & $\mathrm{ACO}$ & 1,11 \\
\hline & TPO & 1,05 & & TPO & 1,08 \\
\hline & Macar-4 & 1,06 & & Macar-4 & 1,06 \\
\hline \multirow{7}{*}{ rat99 } & NN & 1,13 & \multirow{7}{*}{$\mathrm{rd} 400$} & $\mathrm{NN}$ & 1,19 \\
\hline & GA & 1,06 & & GA & 1,08 \\
\hline & SA & 1,05 & & SA & 1,10 \\
\hline & TS & 1,03 & & TS & 1,36 \\
\hline & ACO & 1,09 & & ACO & 1,26 \\
\hline & TPO & 1,05 & & TPO & 1,19 \\
\hline & Macar-4 & 1,09 & & Macar-4 & 1,04 \\
\hline \multirow{7}{*}{ kroA100 } & NN & 1,16 & \multirow{7}{*}{ pcb442 } & $\mathrm{NN}$ & 1,16 \\
\hline & GA & 1,07 & & GA & 1,10 \\
\hline & SA & 1,05 & & SA & 1,13 \\
\hline & TS & 1,06 & & TS & 1,64 \\
\hline & ACO & 1,08 & & ACO & 1,25 \\
\hline & TPO & 1,06 & & TPO & 1,20 \\
\hline & Macar-4 & 1,01 & & Macar-4 & 1,05 \\
\hline \multirow{7}{*}{ eil101 } & NN & 1,17 & & & \\
\hline & GA & 1,09 & & & \\
\hline & SA & 1,07 & & & \\
\hline & TS & 1,06 & & & \\
\hline & ACO & 1,20 & & & \\
\hline & TPO & 1,07 & & & \\
\hline & Macar-4 & 1,00 & & & \\
\hline
\end{tabular}

Analizler bölümünde ortaya çlkan sonuçlar incelendiğinde önerilen yaklaşımın çözüm kalitesi bakımından optimal çözümden sapma değerinin tüm problemler için yaklaşık \%5 civarında olduğu görülmektedir. Çözüm hızı olarak önerilen yaklaşım tartışmasız bir şekilde karşılaştırılan tüm yöntemlerle açık ara üstünlüğe sahiptir. Buradan hareketle önerilen yöntemin hem çözüm performansı hem de çözüm hızı açısından oldukça rekabetçi ve gelecek vadeden bir yaklaşım olabileceği ileri sürülebilir. Elde edilen sonuçlara rağmen akademik olarak konu halen tartışmaya açık durumdadır. Ancak endüstriyel uygulamalar ve/veya teorik araştırmaların farklı alanlarında uygulama firsatları ortaya koyması boyutu ile önerilen yaklaşım dikkate değer sonuçlar üretmiştir. Çünkü optimalden yaklaşık \%5 sapma ile bu kadar hızlı çözümlere erişmek birçok alanda çok kritik sonuçlar elde edilmesini sağlayabilir.

Gelecekte yapılacak çalışmalarda bu yaklaşım akademik anlamda da veri tabanı yönetim sistemleri, büyük veri çalışmaları ya da GSP'nin farklı şekillerde ele alınan problemleri için yeni bir yaklaşım olarak önerilebilir. 
Tablo 7. Büyük GSP Test Problemleri Karşılaştırması

\begin{tabular}{|c|c|c|c|c|c|c|}
\hline \multirow[b]{2}{*}{ Problem } & \multirow[b]{2}{*}{ Optimal } & \multicolumn{2}{|c|}{ Karınca Kolonisi Optimizasyon Algoritması } & \multicolumn{3}{|c|}{ Macar-4 } \\
\hline & & Ortalama Sapma (\%) & Ortalama Süre (sn) & Ortalama Sapma (\%) & Ortalama Süre (sn) & Çözüm \\
\hline pcb442 & 50778 & 3,87 & 44,67 & 4,92 & 0,70 & 53274 \\
\hline d657 & 48912 & 4,45 & 83,97 & 4,63 & 1,82 & 51177 \\
\hline rat783 & 8806 & 5,20 & 110,43 & 5,59 & 3,18 & 9298 \\
\hline pr1002 & 259045 & 5,56 & 170,48 & 6,36 & 3,04 & 275532 \\
\hline pr2392 & 378032 & 7,47 & 834,08 & 5,79 & 22,44 & 399934 \\
\hline Ortalama & 149115 & 5,31 & 248,73 & 5,46 & 6,23 & 157843 \\
\hline pcb3038* & 137694 & & & 5,68 & 160 & 145520 \\
\hline
\end{tabular}

* : Bu problemin çözümü ilgili makalede bulunmamaktadır.

\section{Teşekkür}

Bu çalışma TÜBİTAK TEYDEP 1507 KOBİ AR-GE Başlangıç Destek Programı çerçevesinde gerçekleştirilen 7180837 nolu ve "Lojistik Maliyetlerin Sektör Bazlı Hibrit Uygulamalar İle İyileștirilmesi" adlı proje kapsamında desteklenmiştir. TÜBİTAK' a katkı ve desteklerinden dolayı teşekkür ederim.

\section{Conflict of Interest / Çıkar Çatıșması}

Yazar tarafından herhangi bir çıkar çatışması beyan edilmemiștir.

No conflict of interest was declared by the author.

\section{Kaynaklar}

Antosiewicz, M., Koloch, G., Kamiński, B.,2013. Choice of Best Possible Metaheuristic Algorithm for the Travelling Salesman Problem with Limited Computational Time: Quality, Uncertainty and Speed, Journal of Theoretical and Applied Computer Science, vol. 7, no. 1, pp. 46-55.

Balas, E., Christofides, N., 1981. A Restricted LagrangeanApproach to the Traveling Salesman Problem. Mathematical Programming. 21(1), 1946.

Balinski, M. L., Gomory, R. E., 1964. A Primal Method for the Assignment and Transportation Problems. Management Science, 10(3):578-593. http://dx.doi.org/10.1287/mnsc.10.3.578

Basirzadeh, H., 2014. Ones Assignment Method for Solving Traveling Salesman Problem. Journal of Mathematics and Computer Science. Vol. 10, Iss. 4, pp. 258-265.

Bertsekas, D.P., 1981. A New Algorithm for the Assignment Problem. Mathematical Programming. 21: 152. https://doi.org/10.1007/BF01584237
Burkard, R.E., 1979. Travelling Salesman and Assignment Problems: A Survey. Annals of Discrete Mathematics. Vol. 4, pp. 193-215

Chitty, D. M., 2017. Applying ACO To Large Scale TSP Instances. UK Workshop on Computational Intelligence, pp. 104-118. Springer, Cham, 2017

Croes, G. A. (1958). A Method for Solving TravelingSalesman Problems. Operations Research, 6 (6), 791-812.

Dantzig, G.B., Thapa, M.N., 1997. Linear programming 1: introduction. Springer-Verlang New York, USA.

Dorigo, M., Gambardella, L.M., 1997. Ant Colony System: ACooperative Learning Approach to the Traveling Salesman Problem. IEEE Transactions on Evolutionary Computation, 1(1), 53-66.

Frank, A., 2005. On Kuhn's Hungarian Method-A tribute from Hungary.Naval Research Logistics Quarterly. Vol. 52, Iss. 1, pp. 2-5.

Gendreau, M., Laporte, G., Semet, F., 1998. A TabuSearch Heuristic for the Undirected Selective Travelling Salesman Problem. European Journal of Operational Research, 106(2-3), 539-545.

Halim, A.H., Ismail, I., 2017. Combinatorial Optimization: Comparison of Heuristic Algorithms in Travelling Salesman Problem, Archives of Computational Methods in Engineering, 1-14.

https://doi.org/10.1002/net.3230200605

Jewell, W.S.,1977. The Analytic Methods of Operations Research. Phil. Trans. R. Soc. Lond. A., 287, 373404.

Joines, A., Kay, M.G., Karabacak, M.F., Karagül, K., Tokat, S. Performance analysis of Genetic Algorithm Optimization Toolbox via Traveling Salesperson Problem. Editor: Sayers W. 
Contemporary Issues in Social Sciences and Humanities, 213-221, Landon, UK, AGP Research, 2017.

Jonker, R., Volgenant, T., 1986. Improving the Hungarian Assignment Algorithm. Operations Research Letters. Vol. 5, Iss. 4, pp. 171-175.

Karagul, K., Aydemir, E., Tokat, S., 2016. Using 2-Opt Based Evolution Strategy for Travelling Salesman Problem.An International Journal of Optimization and Control: Theories \& Applications (IJOCTA), 6(2), 103-113.

Kolinski, A., Kolinski, M., 2013. The Use of Hungarian Method in the Evaluation of Production Efficiency (Chapter) in (Eds. RyszardKnosala) Innovations in Management and Production Engineering. Publishing House of Polish Association for Production Management.

Kuhn, H. W., 1955. The Hungarian method for the Assignment Problem. Naval Research Logistics Quarterly. Volume:2, Issue:1-2, s.83-97

Kuhn, H.W., 1956. Variants of the Hungarian Method for Assignment Problems. Naval Research Logistics Quarterly. Volume:3, Issue:4, p. 253-258.

Lawler, E.L., 1971. A solvable case of the traveling salesman problem. Mathematical Programming. Vol. 1, Iss. 1, pp. 267-269.

Little,J.D.C., Murty, K.G., Sweeney, D.W.,Karel, C., 1963. An Algorithm for the Traveling Salesman Problem.Operations Research.Vol. 11, No. 6, pp. 972-989

Lucena, A., 1990. Time-dependent traveling salesman problem-the deliveryman case. Networks. Vol. 20, Iss. 6, pp. 753-763.

Malek, M., Guruswamy, M., Pandya, M., Owens, H., 1989. Serial and Parallel Simulated Annealing andTabu Search Algorithms for the Traveling Salesman Problem.Annals of Operations Research, 21(1), 59-84.

Martello, S., 2010. Jenő Egerváry: From the Origins of the Hungarian Algorithm to Satellite Communication. Cent Eur J Oper Res (2010) 18: 47. https://doi.org/10.1007/s10100-009-0125-z

Mavrovouniotis, M., Yang, S., 2013. Ant Colony Optimization with Immigrants Schemes for the Dynamic Travelling Salesman Problem with Traffic
Factors. Applied Soft Computing. 13(10), 4023 4037.

Mondal,R. N., Hossain, M. R., Saha, S. K., 2013. An Approach for Solving Traveling Salesman Problem. International Journal of Applied Operational Research. Vol. 3, No. 2, pp. 15-26.

Munkres, J., 1957. Algorithms for the Assignment and Transportation Problems. Journal of the Society for Industrial and Applied Mathematics. Vol. 5, No. 1, pp. 32-38.

Nayak, J., Nanda, S., Acharya, S., 2017. Hungarian Method to Solve Travelling Salesman Problem with Fuzzy Cost. International Journal of Mathematics Trends and Technology. (IJMTT) -Vol. 49, Num. 5, pp. 281-284.

P. C. Gilmore, R. E. Gomory, 1964. Sequencing a One State-Variable Machine: A Solvable Case of the Traveling Salesman Problem. Operations Research. vol. 12, Iss. 5, pp. 655-679. http://dx.doi.org/10.1287/opre.12.5.655

Ratliff, H.D., Rosenthal, A.S., 1983. Order Picking in a Rectangular Warehouse: A Solvable Case of the Traveling Salesman Problem. Operations Research, 31 (3), 507-521.

Robinson, J., 1949. On the Hamiltonian Game (A Travelling Salesman Problem). U.S. Air Force Project RAND. RAND Doc. No:204961.

Şahin, Y., Kulak, O., 2013. Depo Operasyonlarının Planlanması İçin Genetik Algoritma Esaslı Modeller. Uluslararası Alanya İşletme Fakültesi Dergisi, 5(3), 141-153.

Şahin, Y., Karagül, K., 2019. Solving Travelling Salesman Problem Using Hybrid Fluid Genetic Algorithm (HFGA), Pamukkale University Journal of Engineering Sciences, Ahead of Print: PAJES81084 | DOI: 10.5505/pajes.2018.81084.

Universität Heidelberg. "Index of / software/TSPLIB95 /tsp". http://comopt.ifi.uni-heidelberg.de/ software/TSPLIB95/tsp/ (18.11.2018).

Winston, W. L., 2003. Operations Research: Applications and Algorithms, Cengage Learning, 4th edition.

Zhao, F., Li, S., Sun, J., Mei, D., 2009. Genetic Algorithm for the One-Commodity Pickup-and-Delivery Traveling Salesman Problem. Computers \& Industrial Engineering, 56(4), 1642-1648. 


\section{Ekler}

Ek-1: Örnek küçük problem için koordinatlar ve uzaklık matrisi

\begin{tabular}{|c|c|c|c|c|c|c|c|c|c|c|c|c|c|c|c|c|c|c|c|c|c|c|}
\hline \multicolumn{2}{|c|}{ [ Koordinatlar] } & \multicolumn{21}{|c|}{ [ Uzaklık Matrisi ] } \\
\hline $\mathbf{x}$ & $\mathbf{Y}$ & & 1 & 2 & 3 & 4 & 5 & 6 & 7 & 8 & 9 & 10 & 11 & 12 & 13 & 14 & 15 & 16 & 17 & 18 & 19 & 20 \\
\hline 87,951292 & 2,658162 & 1 & 0 & 84 & 51 & 81 & 111 & 68 & 31 & 56 & 117 & 48 & 62 & 69 & 80 & 90 & 26 & 55 & 44 & 71 & 69 & 60 \\
\hline 33,466597 & 66,682943 & 2 & 84 & 0 & 60 & 23 & 28 & 65 & 56 & 35 & 34 & 38 & 22 & 17 & 52 & 6 & 67 & 29 & 44 & 18 & 52 & 66 \\
\hline 91,778314 & 53,807184 & 3 & 51 & 60 & 0 & 72 & 87 & 88 & 26 & 55 & 85 & 45 & 48 & 54 & 88 & 66 & 56 & 43 & 23 & 58 & 19 & 83 \\
\hline 20,526749 & 47,633290 & 4 & 81 & 23 & 72 & 0 & 35 & 45 & 59 & 25 & 49 & 34 & 24 & 18 & 29 & 24 & 59 & 30 & 51 & 14 & 69 & 47 \\
\hline 9,006012 & 81,185339 & 5 & 111 & 28 & 87 & 35 & 0 & 79 & 84 & 58 & 19 & 64 & 49 & 42 & 61 & 22 & 92 & 56 & 73 & 41 & 77 & 82 \\
\hline 20,032350 & 2,761925 & 6 & 68 & 65 & 88 & 45 & 79 & 0 & 64 & 36 & 94 & 44 & 51 & 51 & 20 & 68 & 42 & 50 & 66 & 48 & 95 & 8 \\
\hline 41,059603 & 32,578509 & 8 & 56 & 35 & 55 & 25 & 58 & 36 & 36 & 0 & 68 & 11 & 15 & 18 & 33 & 40 & 34 & 14 & 32 & 18 & 59 & 34 \\
\hline 18,692587 & 97,015290 & 9 & 117 & 34 & 85 & 49 & 19 & 94 & 88 & 68 & 0 & 71 & 56 & 50 & 77 & 28 & 101 & 63 & 75 & 50 & 71 & 96 \\
\hline 51,658681 & 33,808405 & 10 & 48 & 38 & 45 & 34 & 64 & 44 & 26 & 11 & 71 & 0 & 15 & 22 & 44 & 43 & 30 & 9 & 22 & 23 & 51 & 40 \\
\hline 44,563128 & 47,541734 & 11 & 62 & 22 & 48 & 24 & 49 & 51 & 36 & 15 & 56 & 15 & 0 & 7 & 44 & 28 & 45 & 8 & 27 & 11 & 47 & 49 \\
\hline 37,806330 & 50,599689 & 12 & 69 & 17 & 54 & 18 & 42 & 51 & 44 & 18 & 50 & 22 & 7 & 0 & 41 & 22 & 51 & 15 & 34 & 4 & 52 & 50 \\
\hline 9,961241 & 20,337535 & 13 & 80 & 52 & 88 & 29 & 61 & 20 & 68 & 33 & 77 & 44 & 44 & 41 & 0 & 53 & 54 & 46 & 66 & 38 & 91 & 26 \\
\hline 28,186895 & 70,415357 & 14 & 90 & 6 & 66 & 24 & 22 & 68 & 62 & 40 & 28 & 43 & 28 & 22 & 53 & 0 & 73 & 35 & 51 & 22 & 57 & 69 \\
\hline 71,285134 & 43,671987 & 17 & 44 & 44 & 23 & 51 & 73 & 66 & 13 & 32 & 75 & 22 & 27 & 34 & 66 & 51 & 39 & 21 & 0 & 38 & 31 & 61 \\
\hline 34,156316 & 49,113437 & 18 & 71 & 18 & 58 & 14 & 41 & 48 & 46 & 18 & 50 & 23 & 11 & 4 & 38 & 22 & 51 & 17 & 38 & 0 & 56 & 48 \\
\hline 85,201575 & 71,837519 & 19 & 69 & 52 & 19 & 69 & 77 & 95 & 41 & 59 & 71 & 51 & 47 & 52 & 91 & 57 & 70 & 45 & 31 & 56 & 0 & 91 \\
\hline 27,466659 & 1,394696 & 20 & 60 & 66 & 83 & 47 & 82 & 8 & 58 & 34 & 96 & 40 & 49 & 50 & 26 & 69 & 35 & 47 & 61 & 48 & 91 & 0 \\
\hline
\end{tabular}

